\title{
Application of Hybrid Cubic B-Spline Collocation Approach for Solving a Generalized Nonlinear Klien-Gordon Equation
}

\author{
Shazalina Mat Zin, ${ }^{1,2}$ Ahmad Abd Majid, ${ }^{1}$ \\ Ahmad Izani Md. Ismail, ${ }^{1}$ and Muhammad Abbas ${ }^{3}$ \\ ${ }^{1}$ School of Mathematical Sciences, Universiti Sains Malaysia, 11800 Pulau Pinang, Malaysia \\ ${ }^{2}$ Institute of Engineering Mathematics, Universiti Malaysia Perlis, 02600 Pauh, Perlis, Malaysia \\ ${ }^{3}$ Department of Mathematics, University of Sargodha, Sargodha 40100, Pakistan
}

Correspondence should be addressed to Shazalina Mat Zin; shazalina@unimap.edu.my

Received 24 June 2014; Accepted 11 December 2014; Published 30 December 2014

Academic Editor: Anuar Ishak

Copyright (c) 2014 Shazalina Mat Zin et al. This is an open access article distributed under the Creative Commons Attribution License, which permits unrestricted use, distribution, and reproduction in any medium, provided the original work is properly cited.

The generalized nonlinear Klien-Gordon equation is important in quantum mechanics and related fields. In this paper, a semiimplicit approach based on hybrid cubic B-spline is presented for the approximate solution of the nonlinear Klien-Gordon equation. The usual finite difference approach is used to discretize the time derivative while hybrid cubic B-spline is applied as an interpolating function in the space dimension. The results of applications to several test problems indicate good agreement with known solutions.

\section{Introduction}

Consider a generalized nonlinear Klien-Gordon (KG) equation in the form of [1]

$$
\begin{array}{r}
u_{t t}+\alpha u_{x x}+\beta u+G(u)=f(x, t), \\
a \leq x \leq b, \quad 0 \leq t \leq T,
\end{array}
$$

subject to the initial conditions

$$
\begin{array}{cc}
u(x, 0)=\omega_{1}(x), & a \leq x \leq b, \\
u_{t}(x, 0)=\omega_{2}(x), & a \leq x \leq b,
\end{array}
$$

and the Dirichlet boundary conditions

$$
\begin{array}{ll}
u(a, t)=\phi_{1}(t), & 0 \leq t \leq T, \\
u(b, t)=\phi_{2}(t), & 0 \leq t \leq T,
\end{array}
$$

where $u(x, t)$ denotes the wave displacement at position $x$ and time $t, G(u)$ is a nonlinear function in $u, \alpha$ and $\beta$ are constants, and $f(x, t), \omega_{1}(x), \omega_{2}(x), \phi_{1}(x)$, and $\phi_{2}(x)$ are known functions.
In the last decade or so, spline functions have been utilized to solve differential equations. For example, Caglar et al. [2] have introduced a cubic B-spline interpolation method to solve two-point boundary value problems. The results obtained were compared to finite difference, finite element, and finite volume method. Caglar et al. [2] concluded that the $\mathrm{B}$-spline interpolation is a better method to interpolate any smooth functions than others. Hamid et al. [3] have developed an alternative cubic trigonometric B-spline interpolation method for the same problem. They have found that the trigonometric B-spline gives better approximation compared to technique used by Caglar et al. Goh et al. [4] have presented a comparison between cubic B-spline and extended cubic B-spline collocation method for solving heat equation. It was concluded that the extended cubic B-spline gives better results. By using the same method, Abbas et al. [5] have solved coupled reaction diffusion system. They found that the B-spline function approximates the system very well and the results are in good agreement with known solutions.

Great deals of research on solving KG equation have been carried out and the results can be found in [6-13]. Dehghan and Shokri [14] have approximated the numerical solution of the nonlinear KG equation using thin plate splines (TPS) 
radial basis functions. The implementation of the method has been claimed to be simple as the finite difference method and the numerical results obtained were more accurate than others in literature. Khuri and Sayfy [1] have solved the generalized nonlinear KG equation using a finite element collocation approach based on third degree B-spline polynomials. Six examples of nonlinear KG equation including SineGordon equation have been analyzed. The proposed method gives compatible results and better approximation compared to Dehghan and Shokri's method in [14]. Rashidinia et al. [15] have presented a cubic B-spline collocation method for solving linear KG equation. The results show the proposed scheme is effective and accurate.

In this paper, a new approach by combining hybrid cubic $\mathrm{B}$-spline function and central finite difference is proposed to solve the KG equation. The finite difference approach is used for the time derivative and the hybrid cubic collocation method is applied to interpolate the solutions at space dimension. The scheme obtained is analyzed by Von Neumann stability analysis. To show the feasibility and accuracy of the method, three problems are considered. Numerical solutions, absolute errors, maximum error, and order of convergence are calculated.

\section{Temporal Discretization}

Consider a uniform mesh $\Omega$ with grid points $\left(x_{j}, t_{k}\right)$ to discretize the grid region $\Delta=[a, b] \times[0, T]$ with $x_{j}=$ $a+j h$ and $t_{k}=k \Delta t$, where $j=0,1,2, \ldots, n$ and $k=$ $0,1,2,3, \ldots, N$. The values of $h$ and $\Delta t$ denote mesh space size and time step size, respectively. The Klien-Gordon equation is approximated at $t_{k+1}$ th time level as follows [16]:

$$
\frac{u_{j}^{k+1}-2 u_{j}^{k}+u_{j}^{k-1}}{(\Delta t)^{2}}+(1-\theta) g_{j}^{k}+\theta g_{j}^{k+1}+G\left(u_{j}^{k}\right)=f_{j}^{k},
$$

where $g_{j}^{k}=\alpha\left(u_{x x}\right)_{j}^{k}+\beta(u)_{j}^{k}, g_{j}^{k+1}=\alpha\left(u_{x x}\right)_{j}^{k+1}+\beta(u)_{j}^{k+1}, 0 \leq$ $\theta \leq 1$, and the subscripts $k$ and $k+1$ are successive time levels. Central difference approach has been used to discretize the time derivative. In order to produce Crank-Nicolson scheme, $\theta$ is chosen to be 0.5 . Hence, the scheme becomes

$$
\begin{aligned}
u_{j}^{k+1} & +0.5(\Delta t)^{2} g_{j}^{k+1} \\
& =2 u_{j}^{k}-0.5(\Delta t)^{2} g_{j}^{k}+(\Delta t)^{2}\left[f_{j}^{k}-G\left(u_{j}^{k}\right)\right]-u_{j}^{k-1} .
\end{aligned}
$$

At $k=0$, there is term, $u_{j}^{-1}$, that is outside of domain. Therefore, initial condition (2b) is approximated by the following central difference approach. Thus,

$$
u_{j}^{-1}=u_{j}^{1}-2 \Delta t \omega_{2}(x) \text {. }
$$

The whole scheme is solved numerically by substituting hybrid cubic B-spline function discussed in next section for $j=0,1, \ldots, n$ at each time level $k$.

\section{Hybrid Cubic B-Spline Collocation Method}

Through this section, hybrid cubic B-spline (HCuBS) is used to solve nonlinear KG equation. The approximate solution, $u(x, t)$, to the analytical solution, $\bar{u}(x, t)$, is considered as

$$
u(x, t)=\sum_{j=-3}^{n-1} C_{j}(t) H_{4, j}(x),
$$

where $C_{j}(t)$ are time-dependent unknowns to be determined and $H_{4, j}(x)$ is hybrid cubic B-spline basis function of order 4 as

$$
H_{4, j}(x)=\gamma B_{4, j}(x)+(1-\gamma) T_{4, j}(x),
$$

where $B_{4, j}(x)$ is cubic $\mathrm{B}$-spline basis function given as

$$
\begin{aligned}
& B_{4, j}(x)
\end{aligned}
$$

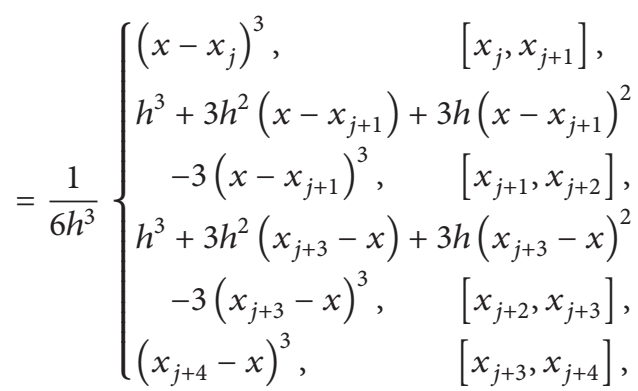

and $T_{4, j}(x)$ is cubic trigonometric B-spline basis function [18, 19 ] given as

$$
\begin{aligned}
& T_{4, j}(x) \\
& =\frac{1}{\mathcal{L}}\left\{\begin{array}{cc}
p^{3}\left(x_{j}\right), & x \in\left[x_{j}, x_{j+1}\right], \\
p\left(x_{j}\right)\left(p\left(x_{j}\right) q\left(x_{j+2}\right)+q\left(x_{j+3}\right) p\left(x_{j+1}\right)\right) & \\
+q\left(x_{j+4}\right) p^{2}\left(x_{j+1}\right), & x \in\left[x_{j+1}, x_{j+2}\right], \\
q\left(x_{j+4}\right)\left(p\left(x_{j+1}\right) q\left(x_{j+3}\right)\right. & \left.q\left(x_{j+4}\right) p\left(x_{j+2}\right)\right) \\
+p\left(x_{j}\right) q^{2}\left(x_{j+3}\right), & x \in\left[x_{j+2}, x_{j+3}\right], \\
q^{3}\left(x_{j+4}\right), & x \in\left[x_{j+3}, x_{j+4}\right],
\end{array}\right.
\end{aligned}
$$

with $p\left(x_{j}\right)=\sin \left(\left(x-x_{j}\right) / 2\right), q\left(x_{j}\right)=\sin \left(\left(x_{j}-x\right) / 2\right)$, and $\kappa=\sin (h / 2) \sin (h) \sin (3 h / 2)$. The value of $\gamma$ plays an important role in the hybrid cubic basis function. If $\gamma=0$, the basis function is equal to cubic trigonometric B-spline basis function and if $\gamma=1$, the basis function is equal to cubic B-spline basis function. Hence, this work just considers the value of $0<\gamma<1$.

Due to local support properties of B-spline basis function, there are only three nonzero basis functions; namely, $H_{4, j-3}\left(x_{j}\right), H_{4, j-2}\left(x_{j}\right)$, and $H_{4, j-1}\left(x_{j}\right)$ are included over subinterval $\left[x_{j}, x_{j+1}\right]$. Thus, the approximate solution and its derivatives with respect to $x$ at $\left(x_{j}, t_{k}\right)$ are

$$
\begin{aligned}
u_{j}^{k} & =A_{1} C_{j-3}^{k}+A_{2} C_{j-2}^{k}+A_{1} C_{j-1}^{k}, \\
\left(u_{x}\right)_{j}^{k} & =A_{3} C_{j-3}^{k}-A_{3} C_{j-1}^{k}, \\
\left(u_{x x}\right)_{j}^{k} & =A_{4} C_{j-3}^{k}\left(t_{k}\right)+A_{5} C_{j-2}^{k}\left(t_{k}\right)+A_{4} C_{j-1}^{k}\left(t_{k}\right),
\end{aligned}
$$


where

$$
A_{i}=\gamma \sigma_{i}+(1-\gamma) \eta_{i}, \quad \text { for } i=1,2, \ldots, 5,
$$

with

$$
\begin{gathered}
\sigma_{1}=\frac{1}{6}, \quad \sigma_{2}=\frac{4}{6}, \quad \sigma_{3}=-\frac{1}{2 h}, \quad \sigma_{4}=\frac{1}{h^{2}}, \\
\sigma_{5}=-\frac{2}{h^{2}}, \quad \eta_{1}=\frac{\kappa_{1}^{2}}{\kappa_{2} \kappa_{3}}, \quad \eta_{2}=\frac{2 \kappa_{1}}{\kappa_{3}}, \\
\eta_{3}=\frac{-3}{4 \kappa_{3}}, \quad \eta_{4}=\frac{3\left(\kappa_{1}-2 \kappa_{1}^{3}+\kappa_{3}\right)}{8 \kappa_{1} \kappa_{2} \kappa_{3}}, \\
\eta_{5}=\frac{-3\left(\kappa_{4}+2 \kappa_{1}{ }^{2} \kappa_{2}\right)}{4 \kappa_{1} \kappa_{2} \kappa_{3}},
\end{gathered}
$$

where

$$
\begin{array}{cl}
\kappa_{1}=\sin \left(\frac{h}{2}\right), & \kappa_{2}=\sin (h), \\
\kappa_{3}=\sin \left(\frac{3 h}{2}\right), & \kappa_{4}=\sin (2 h) .
\end{array}
$$

These approximations are substituted into (4) to produce the matrix system of order $(n+1)$ with $(n+3)$ unknown. In order to generate a unique solution, two additional equations are needed in the system. Hence, boundary conditions (2c) and (2d) are approximated as follows:

$$
\begin{aligned}
& u\left(a, t_{k+1}\right)=A_{1} C_{-3}^{k+1}+A_{2} C_{-2}^{k+1}+A_{1} C_{-1}^{k+1}=\phi_{1}\left(t_{k+1}\right), \\
& u\left(b, t_{k+1}\right)=A_{1} C_{n-3}^{k+1}+A_{2} C_{n-2}^{k+1}+A_{1} C_{n-1}^{k+1}=\phi_{2}\left(t_{k+1}\right) .
\end{aligned}
$$

Thus, the resulting system can be written as

$$
\mathbf{M C}^{k+1}=\mathbf{N C}^{k}-\mathbf{P C}^{k-1}+\mathbf{Q},
$$

where

$$
\mathbf{M}=\left(\begin{array}{ccccccccc}
A_{1} & A_{2} & A_{1} & 0 & \cdots & 0 & 0 & 0 & 0 \\
m_{1} & m_{2} & m_{1} & 0 & & & & & 0 \\
0 & m_{1} & m_{2} & m_{1} & & & & & 0 \\
\vdots & & & & \ddots & & & & \vdots \\
0 & & & & & m_{1} & m_{2} & m_{1} & 0 \\
0 & & & & & 0 & m_{1} & m_{2} & m_{1} \\
0 & 0 & 0 & 0 & \cdots & 0 & A_{1} & A_{2} & A_{1}
\end{array}\right),
$$

$$
\mathbf{N}=\left(\begin{array}{ccccccccc}
0 & 0 & 0 & 0 & \cdots & 0 & 0 & 0 & 0 \\
n_{1} & n_{2} & n_{1} & 0 & & & & & 0 \\
0 & n_{1} & n_{2} & n_{1} & & & & & 0 \\
\vdots & & & & \ddots & & & & \vdots \\
0 & & & & & n_{1} & n_{2} & n_{1} & 0 \\
0 & & & & & 0 & n_{1} & n_{2} & n_{1} \\
0 & 0 & 0 & 0 & \cdots & 0 & 0 & 0 & 0
\end{array}\right)
$$

$$
\begin{gathered}
\mathbf{P}=\left(\begin{array}{ccccccccc}
0 & 0 & 0 & 0 & \cdots & 0 & 0 & 0 & 0 \\
A_{1} & A_{2} & A_{1} & 0 & & & & & 0 \\
0 & A_{1} & A_{2} & A_{1} & & & & & 0 \\
\vdots & & & & \ddots & & & & \vdots \\
0 & & & & & A_{1} & A_{2} & A_{1} & 0 \\
0 & & & & & 0 & A_{1} & A_{2} & A_{1} \\
0 & 0 & 0 & 0 & \cdots & 0 & 0 & 0 & 0
\end{array}\right), \\
\mathbf{Q}=\left(\begin{array}{c}
\phi_{1}\left(t_{k+1}\right) \\
(\Delta t)^{2}\left[f_{0}^{k}-G\left(u_{0}^{k}\right)\right] \\
\vdots \\
(\Delta t)^{2}\left[f_{n}^{k}-G\left(u_{n}^{k}\right)\right] \\
\phi_{2}\left(t_{k+1}\right)
\end{array}\right),
\end{gathered}
$$

with $m_{1}=A_{1}+0.5(\Delta t)^{2}\left[\alpha\left(A_{4}\right)+\beta\left(A_{1}\right)\right], m_{2}=A_{2}+0.5(\Delta t)^{2}$ $\left[\alpha\left(A_{5}\right)+\beta\left(A_{2}\right)\right], n_{1}=2 A_{1}-0.5(\Delta t)^{2}\left[\alpha\left(A_{4}\right)+\beta\left(A_{1}\right)\right]$, and $n_{2}=2 A_{2}-0.5(\Delta t)^{2}\left[\alpha\left(A_{5}\right)+\beta\left(A_{2}\right)\right]$.

This tridiagonal matrix system can be solved using Thomas Algorithm repeatedly for $k=0,1, \ldots N$.

\section{Initial State, $\mathbf{C}^{0}$}

The initial vector, $\mathbf{C}^{0}$, is obtained from initial condition (2a) and boundary values of the derivatives of the initial condition as follows $[2,16]$ :

(i) $\left(u_{x}\right)_{j}^{0}=\omega_{1}^{\prime}\left(x_{j}\right)$ for $j=0$,

(ii) $u_{j}^{0}=\omega_{1}\left(x_{j}\right)$ for $j=0,1,2, \ldots, n$,

(iii) $\left(u_{x}\right)_{j}^{0}=\omega_{1}^{\prime}\left(x_{j}\right)$ for $j=n$.

This operation yields $(n+3) \times(n+3)$ matrix system:

$$
\mathrm{AC}^{0}=\mathbf{B},
$$

$$
\left(\begin{array}{ccccccccc}
A_{3} & 0 & -A_{3} & 0 & \cdots & 0 & 0 & 0 & 0 \\
A_{1} & A_{2} & A_{1} & 0 & & & & & 0 \\
0 & A_{1} & A_{2} & A_{1} & & & & & 0 \\
\vdots & & & & \ddots & & & & \vdots \\
0 & & & & & A_{1} & A_{2} & A_{1} & 0 \\
0 & & & & & 0 & A_{1} & A_{2} & A_{1} \\
0 & 0 & 0 & 0 & \cdots & 0 & A_{3} & 0 & -A_{3}
\end{array}\right)
$$




$$
\times\left(\begin{array}{c}
C_{-3}^{0} \\
C_{-2}^{0} \\
C_{-1}^{0} \\
\vdots \\
C_{n-3}^{0} \\
C_{n-2}^{0} \\
C_{n-1}^{0}
\end{array}\right)=\left(\begin{array}{c}
\omega_{1}^{\prime}\left(x_{0}\right) \\
\omega_{1}\left(x_{0}\right) \\
\vdots \\
\omega_{1}\left(x_{n}\right) \\
\omega_{1}^{\prime}\left(x_{n}\right)
\end{array}\right)
$$

The solution of the tridiagonal system is obtained by using the Thomas Algorithm [20].

\section{Von Neumann Stability Analysis}

The growth of error in single Fourier mode is considered as

$$
C_{j}^{k}=\delta^{k} e^{i \eta j h}
$$

where $i=\sqrt{-1}$ and $\eta$ is the mode number. It is known that this method is applicable to linear scheme. Hence, (1) is linearized by assuming all nonlinear terms equal zero [15]. The following equation is obtained after substituting (10) into the linear scheme:

$$
\begin{aligned}
p_{1} C_{j-3}^{k+1}+p_{2} C_{j-2}^{k+1}+p_{1} C_{j-1}^{k+1} & \\
= & p_{3} C_{j-3}^{k}+p_{4} C_{j-2}^{k}+p_{3} C_{j-1}^{k}-A_{1} C_{j-3}^{k-1}-A_{2} C_{j-2}^{k-1} \\
& \quad-A_{1} C_{j-1}^{k-1}
\end{aligned}
$$

where

$$
\begin{gathered}
p_{1}=A_{1}+\theta(\Delta t)^{2}\left(\alpha A_{4}+\beta A_{1}\right), \\
p_{2}=A_{2}+\theta(\Delta t)^{2}\left(\alpha A_{5}+\beta A_{2}\right), \\
p_{3}=2 A_{1}-(1-\theta)(\Delta t)^{2}\left(\alpha A_{4}+\beta A_{1}\right), \\
p_{4}=2 A_{2}-(1-\theta)(\Delta t)^{2}\left(\alpha A_{5}+\beta A_{2}\right) .
\end{gathered}
$$

By substituting (18) into (19), the following characteristic equation is generated:

$$
A \delta^{2}-B \delta+C=0
$$

where $A=p_{1}[2 \cos (\eta h)]+p_{2}, B=p_{3}[2 \cos (\eta h)]+p_{4}$, and $C=A_{1}[2 \cos (\eta h)]+A_{2}$. Based on Routh-Hurwitz criterion, the transformation, $\delta=(1+v) /(1-v)$, is applied to the characteristic equation $[15,21]$. Then, the equation becomes

$$
(A+B+C) v^{2}+2(A-C) v+(A-B+C)=0 .
$$

The necessary and sufficient conditions for $|\delta| \leq 1$ are $A+B+$ $C \geq 0, A-C \geq 0$ and $A-B+C \geq 0$. Thus, the following terms have been proved:

$$
\begin{gathered}
2 A_{1} \cos (\eta h)+A_{2} \geq 0, \\
2\left[A_{1} \beta+A_{4} \alpha\right] \cos (\eta h)+\left[A_{2} \beta+A_{5} \alpha\right] \geq 0 .
\end{gathered}
$$

Hence, this scheme is concluded to be unconditionally stable.

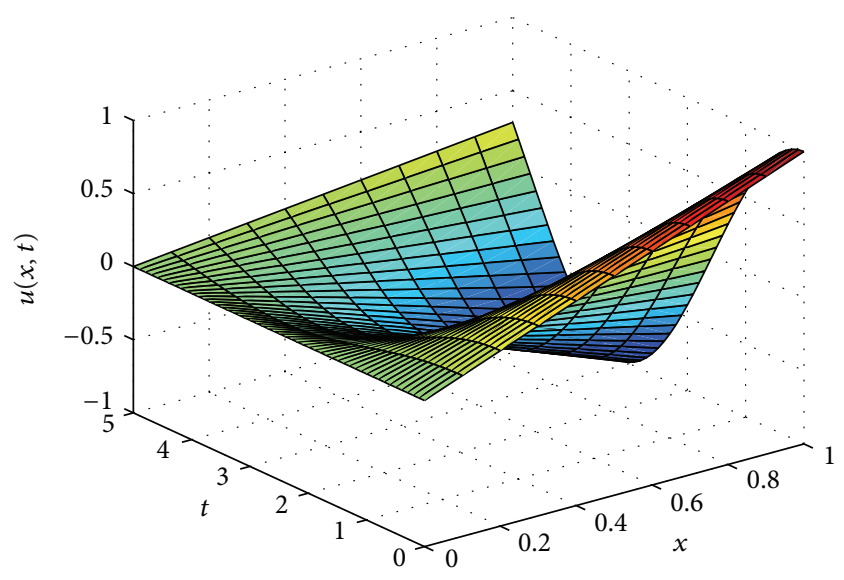

FIGURE 1: Space-time graph for analytical solution of Problem 1.

\section{Numerical Results and Discussions}

In this section, two problems involving KG equation with initial conditions and Dirichlet boundary conditions are tested. In order to measure the accuracy of the method, absolute errors and maximum error are calculated using [22]

$$
\begin{gathered}
\text { Absolute error }=\left|\bar{u}_{i}-u_{i}\right|, \\
\text { Maximum error }=L_{\infty}=\max _{i}\left|\bar{u}_{i}-u_{i}\right|,
\end{gathered}
$$

where $\bar{u}_{i}$ and $u_{i}$ are analytical solution and approximate solution, respectively. The numerical order of convergence, $p$, is obtained by using [1]

$$
p=\frac{\log \left(L_{\infty}(n)\right)-\log \left(L_{\infty}(2 n)\right)}{\log (T / n)-\log (T / 2 n)},
$$

where $L_{\infty}(n)$ and $L_{\infty}(2 n)$ are the $L_{\infty}$ at number of partitions $n$ and $2 n$, respectively.

Problem 1. Consider the following nonlinear Klien-Gordon equation as $[1,14]$

$$
\begin{array}{r}
u_{t t}-u_{x x}+u^{2}=-x \cos t+x^{2} \cos ^{2} t, \\
0 \leq x \leq 1, \quad 0 \leq t \leq 5,
\end{array}
$$

subject to the initial conditions

$$
u(x, 0)=x \quad u_{t}(x, 0)=0
$$

and boundary conditions

$$
u(0, t)=0 \quad u(1, t)=\cos t .
$$

The analytical solution is given by $\bar{u}(x, t)=x \cos t$. Figure 1 shows the space-time plot for this analytical solution.

Initially, this problem is tested by $h=0.1$ and $\Delta t=0.005$. Numerical solutions of this problem at $t=5$ are listed in Table 1. The absolute errors of this problem at the same point are tabulated in Table 2 . The absolute errors at $t=5$ 
TABLE 1: Numerical solution of Problem 1 at $t=5$ with $h=0.1$ and $\Delta t=0.005$.

\begin{tabular}{lcccc}
\hline$x$ & Analytical solution & HCuBS $(\gamma=0.1)$ & HCuBS $(\gamma=0.5)$ & HCuBS $(\gamma=0.9)$ \\
\hline 0.2 & 0.0567 & 0.0567 & 0.0567 & 0.0567 \\
0.4 & 0.1135 & 0.1134 & 0.1134 & 0.1135 \\
0.6 & 0.1702 & 0.1702 & 0.1702 & 0.1702 \\
0.8 & 0.2269 & 0.2269 & 0.2269 & 0.2269 \\
1.0 & 0.2837 & 0.2837 & 0.2837 & 0.2837 \\
\hline
\end{tabular}

TABLe 2: Absolute error of Problem 1 at $t=5$ with $h=0.1$ and $\Delta t=0.005$.

\begin{tabular}{lcccc}
\hline$x$ & Mat Zin et al. [17] & HCuBS $(\gamma=0.1)$ & HCuBS $(\gamma=0.5)$ & HCuBS $(\gamma=0.9)$ \\
\hline 0.2 & $2.473 \times 10^{-5}$ & $2.227 \times 10^{-5}$ & $1.242 \times 10^{-5}$ & $2.571 \times 10^{-6}$ \\
0.4 & $3.810 \times 10^{-5}$ & $3.432 \times 10^{-5}$ & $1.916 \times 10^{-5}$ & $3.969 \times 10^{-6}$ \\
0.6 & $3.559 \times 10^{-5}$ & $3.206 \times 10^{-5}$ & $1.791 \times 10^{-5}$ & $3.714 \times 10^{-6}$ \\
0.8 & $2.152 \times 10^{-5}$ & $1.938 \times 10^{-5}$ & $1.082 \times 10^{-5}$ & $2.243 \times 10^{-6}$ \\
\hline
\end{tabular}

Table 3: Maximum error of Problem 1 compared to Khuri and Sayfy [1], Dehghan and Shokri [14], and Mat Zin et al. [17].

\begin{tabular}{|c|c|c|c|c|c|}
\hline$t$ & 1 & 2 & 3 & 4 & 5 \\
\hline \multicolumn{6}{|l|}{ For $(h=0.02, \Delta t=0.0001)$} \\
\hline Dehghan and Shokri [14] & $1.254 \times 10^{-5}$ & - & $1.555 \times 10^{-5}$ & - & $3.379 \times 10^{-5}$ \\
\hline \multicolumn{6}{|l|}{ For $(h=0.1, \Delta t=0.005)$} \\
\hline Khuri and Sayfy [1] & $2.838 \times 10^{-4}$ & $3.299 \times 10^{-4}$ & $7.055 \times 10^{-5}$ & $3.018 \times 10^{-4}$ & $3.249 \times 10^{-4}$ \\
\hline Mat Zin et al. [17] & $4.552 \times 10^{-5}$ & $4.358 \times 10^{-5}$ & $1.359 \times 10^{-5}$ & $5.353 \times 10^{-5}$ & $3.868 \times 10^{-5}$ \\
\hline $\operatorname{HCuBS}(\gamma=0.1)$ & $4.100 \times 10^{-5}$ & $3.925 \times 10^{-5}$ & $1.224 \times 10^{-5}$ & $4.821 \times 10^{-5}$ & $3.484 \times 10^{-5}$ \\
\hline $\operatorname{HCuBS}(\gamma=0.5)$ & $2.288 \times 10^{-5}$ & $2.190 \times 10^{-5}$ & $6.826 \times 10^{-6}$ & $2.691 \times 10^{-5}$ & $1.946 \times 10^{-5}$ \\
\hline $\operatorname{HCuBS}(\gamma=0.9)$ & $4.739 \times 10^{-6}$ & $4.536 \times 10^{-6}$ & $1.414 \times 10^{-6}$ & $5.575 \times 10^{-6}$ & $4.033 \times 10^{-6}$ \\
\hline
\end{tabular}

TABLe 4: Numerical solution of Problem 1 at $t=5$ with $h=0.04$ and $\Delta t=0.001$.

\begin{tabular}{lcccc}
\hline$x$ & Analytical solution & HCuBS $(\gamma=0.1)$ & HCuBS $(\gamma=0.5)$ & HCuBS $(\gamma=0.9)$ \\
\hline 0.2 & 0.0567 & 0.0567 & 0.0567 & 0.0567 \\
0.4 & 0.1135 & 0.1135 & 0.1135 & 0.1135 \\
0.6 & 0.1702 & 0.1702 & 0.1702 & 0.1702 \\
0.8 & 0.2269 & 0.2269 & 0.2269 & 0.2269 \\
1.0 & 0.2837 & 0.2837 & 0.2837 & 0.2837 \\
\hline
\end{tabular}

with $\gamma=0.1, \gamma=0.5$, and $\gamma=0.9$ are also depicted in Figure 2. It can be seen numerically and graphically that HCuBS with $\gamma=0.9$ is capable of giving better results. This observation is established by comparing the maximum error obtained with the maximum error obtained by Khuri and Sayfy [1], Dehghan and Shokri [14], and Mat Zin et al. [17]. Table 3 tabulates the comparison value of maximum errors at different time levels.

Then, this problem is tested by $h=0.04$ and $\Delta t=0.001$. Tables 4 and 5 illustrate the numerical solutions and absolute errors of this problem at $t=5$, respectively. Graphically, the absolute error of this problem at $t=5$ with $\gamma=0.1, \gamma=$ 0.5 , and $\gamma=0.9$ is plotted in Figure 3. The comparison of maximum error obtained from present method with Khuri and Sayfy [1], Dehghan and Shokri [14], and Mat Zin et al.
[17] methods is shown in Table 6. Clearly from the tables and figures, $\mathrm{HCuBS}$ with $\gamma=0.9$ approximates this problem very well compared to Khuri and Sayfy [1], Dehghan and Shokri [14], and Mat Zin et al. [17].

The order of convergence of the present problem is tabulated in Table 7. An examination of this table indicates that the method has a nearly second order of convergence.

Problem 2. The nonlinear Klien-Gordon equation is considered as [14]

$$
\begin{array}{r}
u_{t t}-\frac{5}{2} u_{x x}+u+\frac{3}{2} u^{3}=0, \\
0 \leq x \leq 1, \quad 0 \leq t \leq 4,
\end{array}
$$


TABle 5: Absolute error of Problem 1 at $t=5$ with $h=0.04$ and $\Delta t=0.001$.

\begin{tabular}{lcccc}
\hline$x$ & Mat Zin et al. [17] & HCuBS $(\gamma=0.1)$ & HCuBS $(\gamma=0.5)$ & HCuBS $(\gamma=0.9)$ \\
\hline 0.2 & $4.404 \times 10^{-6}$ & $3.964 \times 10^{-6}$ & $2.205 \times 10^{-6}$ & $4.450 \times 10^{-7}$ \\
0.4 & $6.172 \times 10^{-6}$ & $5.556 \times 10^{-6}$ & $3.090 \times 10^{-6}$ & $6.237 \times 10^{-7}$ \\
0.6 & $5.745 \times 10^{-6}$ & $5.171 \times 10^{-6}$ & $2.876 \times 10^{-6}$ & $5.805 \times 10^{-7}$ \\
0.8 & $3.524 \times 10^{-6}$ & $3.172 \times 10^{-6}$ & $1.764 \times 10^{-6}$ & $5.560 \times 10^{-7}$ \\
\hline
\end{tabular}

TAble 6: Maximum error of Problem 1 compared to Khuri and Sayfy [1], Dehghan and Shokri [14], and Mat Zin et al. [17].

\begin{tabular}{|c|c|c|c|c|c|}
\hline$t$ & 1 & 2 & 3 & 4 & 5 \\
\hline \multicolumn{6}{|l|}{ For $(h=0.02, \Delta t=0.0001)$} \\
\hline Dehghan and Shokri [14] & $1.254 \times 10^{-5}$ & - & $1.555 \times 10^{-5}$ & - & $3.379 \times 10^{-5}$ \\
\hline \multicolumn{6}{|l|}{ For $(h=0.04, \Delta t=0.001)$} \\
\hline Khuri and Sayfy [1] & $4.599 \times 10^{-5}$ & $8.053 \times 10^{-5}$ & $1.276 \times 10^{-5}$ & $7.292 \times 10^{-5}$ & $5.128 \times 10^{-5}$ \\
\hline Mat Zin et al. [17] & $7.316 \times 10^{-6}$ & $6.986 \times 10^{-6}$ & $2.089 \times 10^{-6}$ & $8.596 \times 10^{-6}$ & $6.245 \times 10^{-6}$ \\
\hline $\operatorname{HCuBS}(\gamma=0.1)$ & $6.585 \times 10^{-6}$ & $6.288 \times 10^{-6}$ & $1.880 \times 10^{-6}$ & $7.738 \times 10^{-6}$ & $5.622 \times 10^{-6}$ \\
\hline $\operatorname{HCuBS}(\gamma=0.5)$ & $3.662 \times 10^{-6}$ & $3.497 \times 10^{-6}$ & $1.046 \times 10^{-6}$ & $4.304 \times 10^{-6}$ & $3.127 \times 10^{-6}$ \\
\hline $\operatorname{HCuBS}(\gamma=0.9)$ & $7.390 \times 10^{-7}$ & $7.057 \times 10^{-7}$ & $2.110 \times 10^{-7}$ & $8.645 \times 10^{-7}$ & $6.311 \times 10^{-7}$ \\
\hline
\end{tabular}

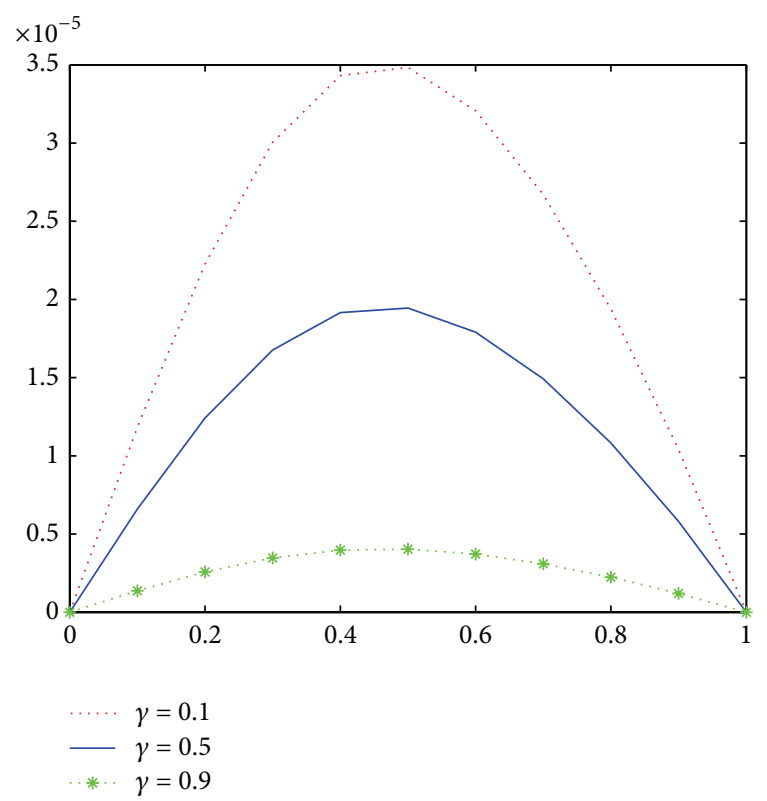

FIGURE 2: Absolute error of Problem 1 with $h=0.1$ and $\Delta t=0.005$.

TABLE 7: The maximum error and order of convergence of Problem 1 at $t=5$.

\begin{tabular}{lccccc}
\hline & $\Delta t=0.005$ & & \multicolumn{3}{c}{$\Delta t=0.001$} \\
$n$ & $L_{\infty}$ & $p$ & $n$ & $L_{\infty}$ & $p$ \\
\hline 5 & $1.538 \times 10^{-5}$ & - & 25 & $6.311 \times 10^{-7}$ & - \\
10 & $4.033 \times 10^{-6}$ & 1.932 & 50 & $1.633 \times 10^{-7}$ & 1.886 \\
20 & $1.116 \times 10^{-7}$ & 1.853 & 100 & $4.603 \times 10^{-8}$ & 1.827 \\
\hline
\end{tabular}

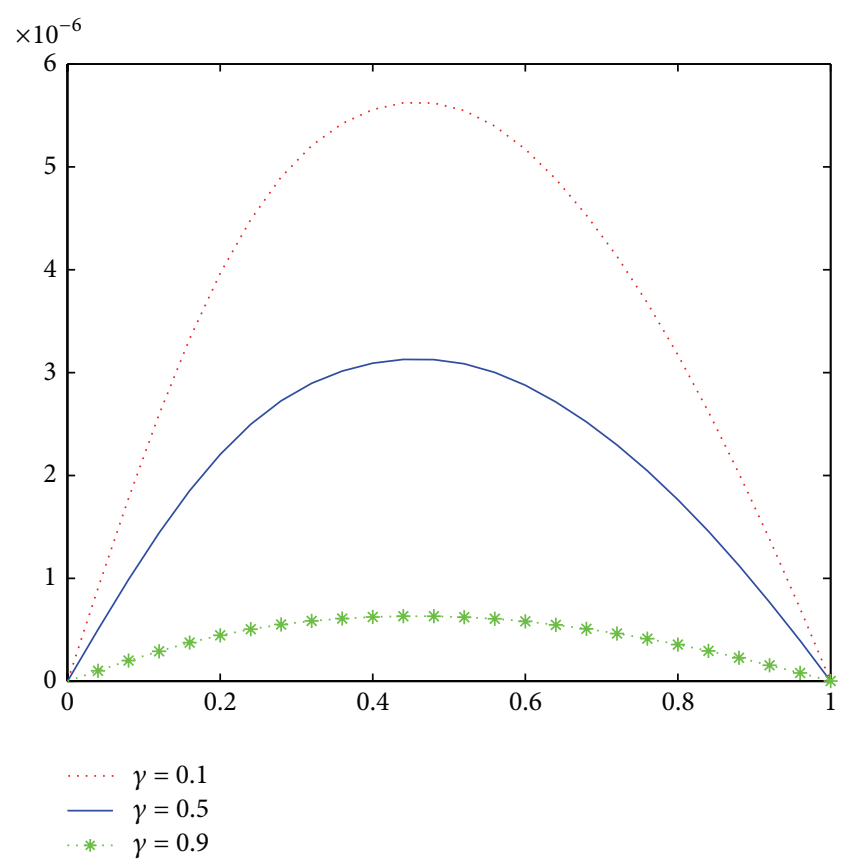

FIgure 3: Absolute error of Problem 1 with $h=0.04$ and $\Delta t=0.001$.

with the initial and boundary conditions

$$
\begin{array}{cc}
u(x, 0)=B \tan (K x), & u_{t}(x, 0)=c B K \sec ^{2}(K x), \\
u(0, t)=B \tan (K c t), & u(1, t)=B \tan [K(1+c t)],
\end{array}
$$


TABLE 8: Numerical solution of Problem 2 at $t=4$ with $h=0.01$ and $\Delta t=0.001$.

\begin{tabular}{lcccc}
\hline$x$ & Analytical solution & HCuBS $(\gamma=0.1)$ & HCuBS $(\gamma=0.5)$ & HCuBS $(\gamma=0.9)$ \\
\hline 0.1 & 1.2439 & 1.2440 & 1.2440 & 1.2440 \\
0.2 & 1.3818 & 1.3819 & 1.3819 & 1.3819 \\
0.3 & 1.5435 & 1.5438 & 1.5438 & 1.5438 \\
0.4 & 1.7369 & 1.7373 & 1.9739 & 1.7373 \\
0.5 & 1.9735 & 1.9739 & 2.2715 & 1.9739 \\
0.6 & 2.2710 & 2.2715 & 2.6588 & 2.2715 \\
0.7 & 2.6583 & 2.6588 & 3.1868 & 2.6588 \\
0.8 & 3.1862 & 3.1867 & 3.9528 & 3.1868 \\
0.9 & 3.9523 & 3.9527 & 5.1718 & 3.9528 \\
1.0 & 5.1718 & 5.1718 & 5.1718 \\
\hline
\end{tabular}

TABLE 9: Absolute error of Problem 2 at $t=4$ with $h=0.01$ and $\Delta t=0.001$.

\begin{tabular}{lccc}
\hline$x$ & HCuBS $(\gamma=0.1)$ & HCuBS $(\gamma=0.5)$ & HCuBS $(\gamma=0.9)$ \\
\hline 0.1 & $8.706 \times 10^{-5}$ & $8.825 \times 10^{-5}$ & $8.944 \times 10^{-5}$ \\
0.2 & $1.699 \times 10^{-4}$ & $1.721 \times 10^{-4}$ & $1.744 \times 10^{-4}$ \\
0.3 & $2.501 \times 10^{-4}$ & $2.533 \times 10^{-4}$ & $2.564 \times 10^{-4}$ \\
0.4 & $3.289 \times 10^{-4}$ & $3.328 \times 10^{-4}$ & $3.368 \times 10^{-4}$ \\
0.5 & $4.065 \times 10^{-4}$ & $4.112 \times 10^{-4}$ & $4.158 \times 10^{-4}$ \\
0.6 & $4.810 \times 10^{-4}$ & $4.861 \times 10^{-4}$ & $4.913 \times 10^{-4}$ \\
0.7 & $5.439 \times 10^{-4}$ & $5.493 \times 10^{-4}$ & $5.547 \times 10^{-4}$ \\
0.8 & $5.695 \times 10^{-4}$ & $5.746 \times 10^{-4}$ & $5.798 \times 10^{-4}$ \\
0.9 & $4.783 \times 10^{-4}$ & $4.822 \times 10^{-4}$ & $4.861 \times 10^{-4}$ \\
\hline
\end{tabular}

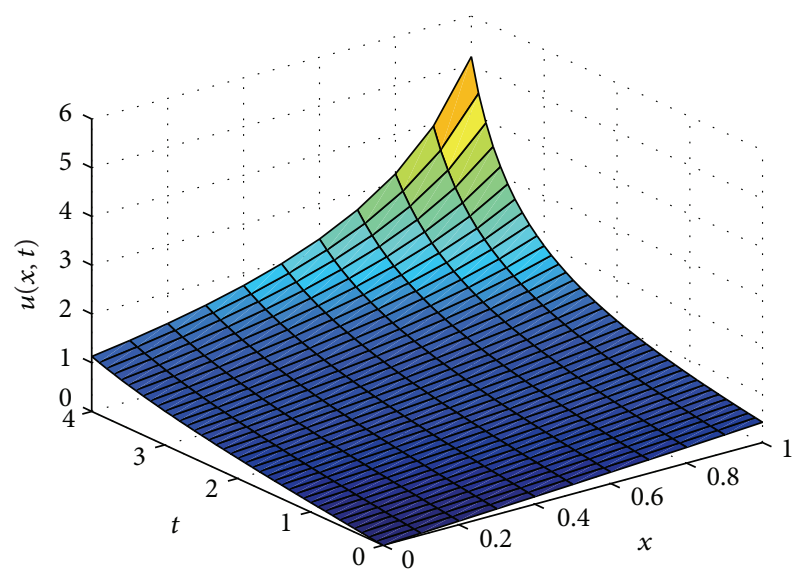

FIgURE 4: Space-time graph for analytical solution of Problem 2.

where $B=\sqrt{2 / 3}, K=\sqrt{-1 /\left(-5+c^{2}\right)}$, and $c=0.5$. The analytical solution is known to be $\bar{u}(x, t)=B \tan [K(x+c t)]$. Space-time plot for this analytical solution is shown in Figure 4.

This problem is solved numerically using $h=0.01$ and $\Delta t=0.001$. Tables 8 and 9 list approximate solutions and absolute errors at $t=4$, respectively. The graphical plot of absolute errors at $t=4$ with three different values of $\gamma$ is shown in Figure 5. The maximum error of this problem is

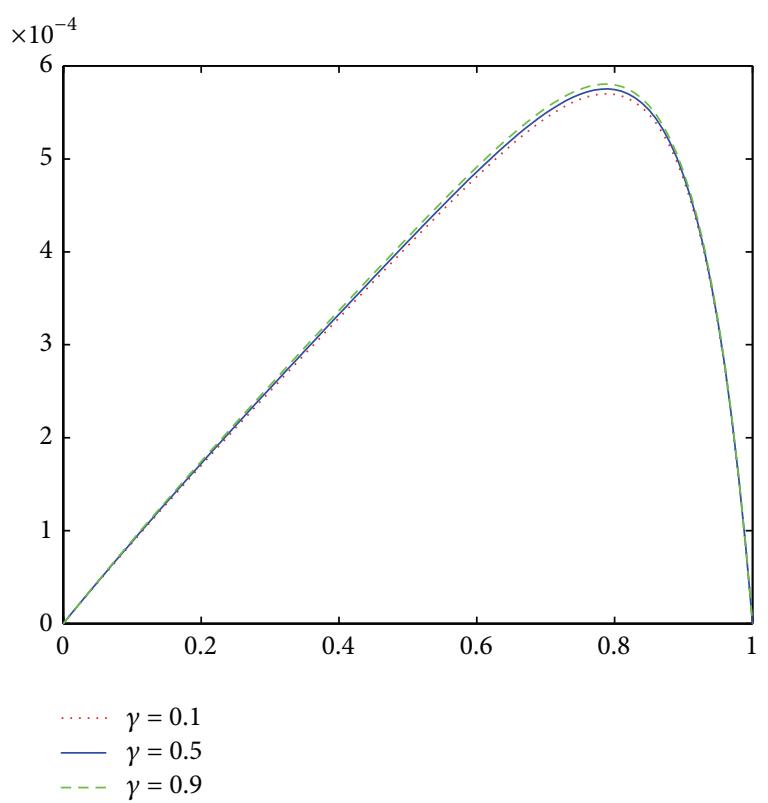

Figure 5: Absolute error of Problem 2 with $h=0.01$ and $\Delta t=0.001$.

compared with Dehghan and Shokri [14] work which is listed in Table 10. The tables indicate that HCuBS with $\gamma=0.1$ gives better results to this problem. 
TABLE 10: Maximum error of Problem 2 compared to Dehghan and Shokri [14] with $h=0.01$ and $\Delta t=0.001$.

\begin{tabular}{lcccc}
\hline$t$ & 1 & 2 & 3 & 4 \\
\hline Dehghan and Shokri $[14]$ & $5.996 \times 10^{-6}$ & $2.197 \times 10^{-5}$ & $9.089 \times 10^{-5}$ & $8.295 \times 10^{-4}$ \\
HCuBS $(\gamma=0.1)$ & $4.878 \times 10^{-6}$ & $1.871 \times 10^{-5}$ & $7.613 \times 10^{-5}$ & $5.701 \times 10^{-4}$ \\
HCuBS $(\gamma=0.5)$ & $5.189 \times 10^{-6}$ & $1.947 \times 10^{-5}$ & $7.775 \times 10^{-5}$ & $5.753 \times 10^{-4}$ \\
HCuBS $(\gamma=0.9)$ & $5.500 \times 10^{-6}$ & $2.024 \times 10^{-5}$ & $7.938 \times 10^{-5}$ & $5.805 \times 10^{-4}$ \\
\hline
\end{tabular}

TABLE 11: Numerical solution of Problem 3 at $t=1$ with $h=(\ln 2) / 5$ and $\Delta t=0.01$.

\begin{tabular}{lcccc}
\hline$x$ & Analytical solution & HCuBS $(\gamma=0.1)$ & HCuBS $(\gamma=0.5)$ & HCuBS $(\gamma=0.9)$ \\
\hline$(\ln 2) / 5$ & 3.1224 & 3.1221 & 3.1220 & 3.1219 \\
$2(\ln 2) / 5$ & 3.0657 & 3.0653 & 3.0650 & 3.0650 \\
$3(\ln 2) / 5$ & 2.9736 & 2.9732 & 2.9731 & 2.9729 \\
$4(\ln 2) / 5$ & 2.8497 & 2.8495 & 2.8494 & 2.8493 \\
$\ln 2$ & 2.6690 & 2.6690 & 2.6690 & 2.6990 \\
\hline
\end{tabular}

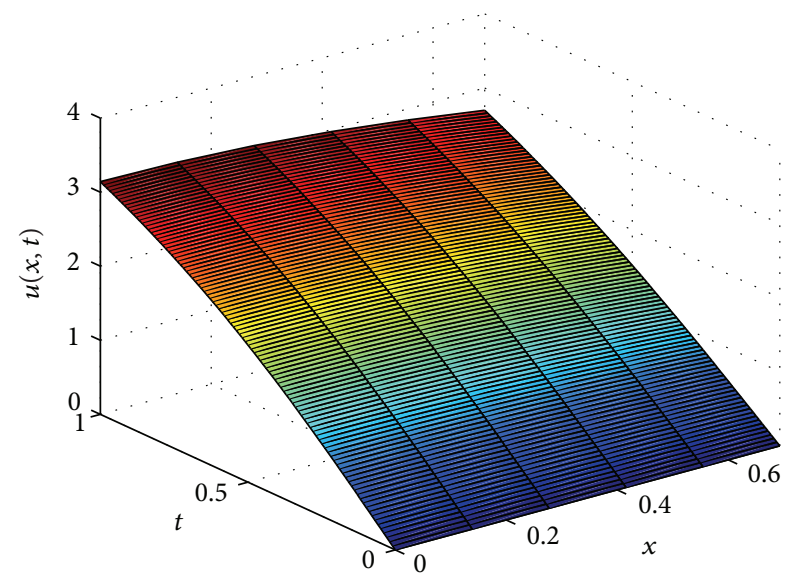

Figure 6: Space-time graph for analytical solution of Problem 3.

Problem 3. Let the nonlinear Klien-Gordon equation be considered as [1]

$$
u_{t t}-u_{x x}+\sin u=0, \quad 0 \leq x \leq \ln 2,0 \leq t \leq 1 .
$$

This equation is also known as Sine-Gordon equation. The initial and boundary conditions are given by

$$
\begin{gathered}
u(x, 0)=0, \quad u_{t}(x, 0)=4 \operatorname{sech} x \\
u(0, t)=4 \tan ^{-1} t, \quad u(\ln 2, t)=4 \tan ^{-1}\left(\frac{4 t}{5}\right) .
\end{gathered}
$$

The analytical solution of this problem is given as $\bar{u}(x, t)=$ $4 \tan ^{-1}(t \operatorname{sech} x)$. Figure 6 presents the space-time graph of this analytical solution.

HCuBS collocation method is used to solve this problem numerically with parameters $h=(\ln 2) / 5$ and $\Delta t=0.01$. Table 11 tabulates the approximate solutions of this problem at $t=1$ and Table 12 tabulates the absolute errors of this problem also at $t=1$. The graphical comparison of the absolute errors with different value of $\gamma$ at $t=1$ is depicted in Figure 7.

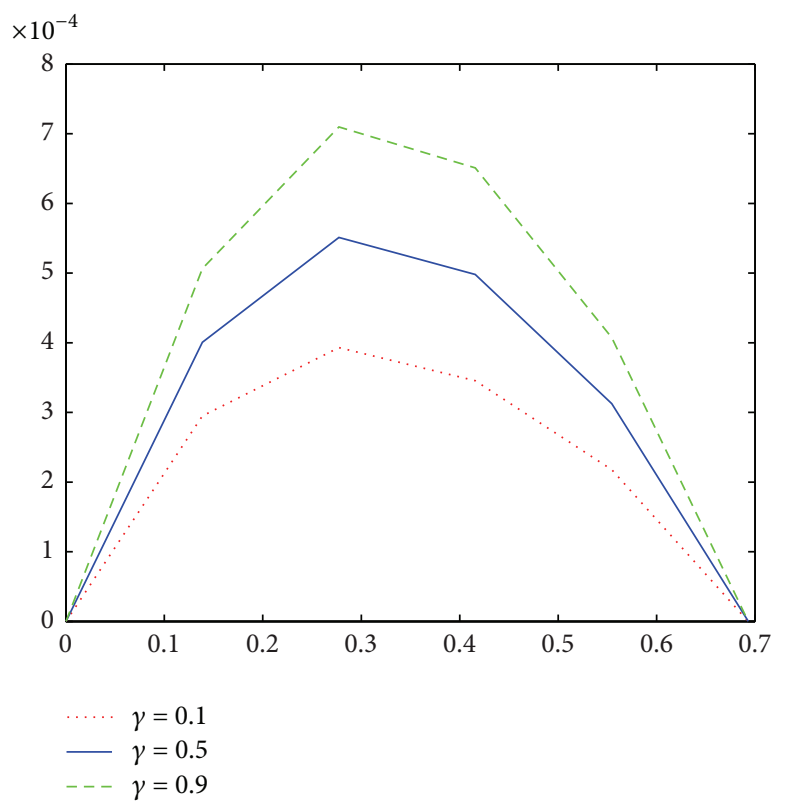

Figure 7: Absolute error of Problem 3 with $h=(\ln 2) / 5$ and $\Delta t=$ 0.01 .

The maximum errors of this problem are compared with Khuri and Sayfy [1] and Mat Zin et al. [17] work (Table 13). The table shows that Mat Zin et al. produce better results which are the errors just slightly better than HCuBS with $\gamma=0.1$.

\section{Conclusion}

In this work, the generalized Klien-Gordon equation was successfully solved using HCuBS collocation method. Specifically, the central difference approach has been applied to discretize the time derivative and hybrid cubic B-spline function had been used to interpolate the solution in space dimension. Three problems have been tested using the proposed method and the solutions obtained were in good agreement with 
TABLE 12: Absolute error of Problem 3 at $t=1$ with $h=(\ln 2) / 5$ and $\Delta t=0.01$.

\begin{tabular}{lcccc}
\hline$x$ & Mat Zin et al. [17] & HCuBS $(\gamma=0.1)$ & HCuBS $(\gamma=0.5)$ & HCuBS $(\gamma=0.9)$ \\
\hline$(\ln 2) / 5$ & $2.686 \times 10^{-4}$ & $2.950 \times 10^{-4}$ & $4.006 \times 10^{-4}$ & $5.064 \times 10^{-4}$ \\
$2(\ln 2) / 5$ & $3.534 \times 10^{-4}$ & $3.929 \times 10^{-4}$ & $5.511 \times 10^{-4}$ & $7.097 \times 10^{-4}$ \\
$3(\ln 2) / 5$ & $3.072 \times 10^{-4}$ & $3.453 \times 10^{-4}$ & $4.980 \times 10^{-4}$ & $6.509 \times 10^{-4}$ \\
$4(\ln 2) / 5$ & $1.942 \times 10^{-4}$ & $2.179 \times 10^{-4}$ & $3.125 \times 10^{-4}$ & $4.072 \times 10^{-4}$ \\
\hline
\end{tabular}

TABle 13: Maximum error of Problem 3 compared to Khuri and Sayfy [1] and Mat Zin et al. [17] with $h=(\ln 2) / 5$ and $\Delta t=0.01$.

\begin{tabular}{lccccc}
\hline$t$ & 0.01 & 0.02 & 0.10 & 0.50 & 1.00 \\
\hline Khuri and Sayfy [1] & $1.3 \times 10^{-6}$ & $6.6 \times 10^{-6}$ & $3.8 \times 10^{-4}$ & $4.8 \times 10^{-3}$ & $2.2 \times 10^{-3}$ \\
Mat Zin et al. [17] & $5.566 \times 10^{-7}$ & $1.618 \times 10^{-6}$ & $9.851 \times 10^{-5}$ & $1.323 \times 10^{-3}$ & $3.534 \times 10^{-4}$ \\
HCuBS $(\gamma=0.1)$ & $5.565 \times 10^{-7}$ & $1.621 \times 10^{-6}$ & $9.876 \times 10^{-5}$ & $1.323 \times 10^{-3}$ & $3.929 \times 10^{-4}$ \\
HCuBS $(\gamma=0.5)$ & $5.560 \times 10^{-7}$ & $1.632 \times 10^{-6}$ & $9.978 \times 10^{-5}$ & $1.422 \times 10^{-3}$ & $5.511 \times 10^{-4}$ \\
HCuBS $(\gamma=0.9)$ & $5.554 \times 10^{-7}$ & $1.644 \times 10^{-6}$ & $1.008 \times 10^{-4}$ & $1.464 \times 10^{-3}$ & $7.097 \times 10^{-4}$ \\
\hline
\end{tabular}

the analytical solution. Through this method, an accurate solution at an intermediate point can be easily calculated.

\section{Conflict of Interests}

The authors declare that there is no conflict of interests regarding the publication of this paper.

\section{Acknowledgment}

The authors acknowledge financial support by Fundamental Research Grant Scheme (FRGS) with no. 203/PMATHS/ 6711324 from School of Mathematical Sciences, Universiti Sains Malaysia (USM), Penang, Malaysia.

\section{References}

[1] S. A. Khuri and A. Sayfy, "A spline collocation approach for the numerical solution of a generalized nonlinear Klein-Gordon equation," Applied Mathematics and Computation, vol. 216, no. 4, pp. 1047-1056, 2010.

[2] H. Caglar, N. Caglar, and K. Elfaituri, "B-spline interpolation compared with finite difference, finite element and finite volume methods which applied to two-point boundary value problems," Applied Mathematics and Computation, vol. 175, no. 1, pp. 72-79, 2006.

[3] N. N. Hamid, A. A. Majid, and A. I. M. Ismail, "Cubic trigonometric B-spline applied to linear two-point boundary value problems of order two," World Academic of Science, Engineering and Technology, no. 47, pp. 478-803, 2010.

[4] J. Goh, A. A. Majid, and A. I. Ismail, "A comparison of some splines-based methods for the one-dimensional heat equation," Proceedings of World Academy of Science, Engineering and Technology, vol. 7, no. 47, pp. 858-861, 2010.

[5] M. Abbas, A. A. Majid, A. I. Md Ismail, and A. Rashid, "Numerical method using cubic B-spline for a strongly coupled reaction-diffusion system," PLOS ONE, vol. 9, no. 1, Article ID e83265, 2014.

[6] W. M. Cao and B. Y. Guo, "Fourier collocation method for solving nonlinear Klein-Gordon equation," Journal of Computational Physics, vol. 108, no. 2, pp. 296-305, 1993.
[7] E. Y. Deeba and S. A. Khuri, "A decomposition method for solving the nonlinear Klein-Gordon equation," Journal of Computational Physics, vol. 124, no. 2, pp. 442-448, 1996.

[8] G. Ben-Yu, L. Xun, and L. Vázquez, "A Legendre spectral method for solving the nonlinear Klein-Gordon equation," Computational and Applied Mathematics, vol. 15, no. 1, pp. 19-36, 1996.

[9] Y. S. Wong, Q. Chang, and L. Gong, "An initial-boundary value problem of a nonlinear Klein-Gordon equation," Applied Mathematics and Computation, vol. 84, no. 1, pp. 77-93, 1997.

[10] A.-M. Wazwaz, "The tanh and the sine-cosine methods for compact and noncompact solutions of the nonlinear KleinGordon equation," Applied Mathematics and Computation, vol. 167, no. 2, pp. 1179-1195, 2005.

[11] Sirendaoreji, "Auxiliary equation method and new solutions of Klein-Gordon equations," Chaos, Solitons \& Fractals, vol. 31, no. 4, pp. 943-950, 2007.

[12] U. Yücel, "Homotopy analysis method for the sine-Gordon equation with initial conditions," Applied Mathematics and Computation, vol. 203, no. 1, pp. 387-395, 2008.

[13] M. S. Chowdhury and I. Hashim, "Application of homotopyperturbation method to Klein-GORdon and sine-GORdon equations," Chaos, Solitons and Fractals, vol. 39, no. 4, pp. 19281935, 2009.

[14] M. Dehghan and A. Shokri, "Numerical solution of the nonlinear Klein-Gordon equation using radial basis functions," Journal of Computational and Applied Mathematics, vol. 230, no. 2, pp. 400-410, 2009.

[15] J. Rashidinia, F. Asfahani, and S. Jamalzadeh, "B-spline collocation approach for solution of Klien-Gordon equation," International Journal of Mathematical Modelling and Computations, vol. 3, no. 1, pp. 25-33, 2013.

[16] I. Dağ, D. Irk, and B. Saka, "A numerical solution of the Burgers' equation using cubic B-splines," Applied Mathematics and Computation, vol. 163, no. 1, pp. 199-211, 2005.

[17] S. Mat Zin, M. Abbas, A. Abd Majid, and A. I. Md Ismail, "A new trigonometric spline approach to numerical solution of generalized nonlinear klien-gordon equation," PLoS ONE, vol. 9, no. 5, Article ID e95774, 2014.

[18] M. Abbas, A. A. Majid, A. I. Ismail, and A. Rashid, "The application of cubic trigonometric B-spline to the numerical solution of the hyperbolic problems," Applied Mathematics and Computation, vol. 239, pp. 74-88, 2014. 
[19] M. Abbas, A. A. Majid, A. I. Ismail, and A. Rashid, "Numerical method using cubic trigonometric B-spline technique for nonclassical diffusion problems," Abstract and Applied Analysis, vol. 2014, Article ID 849682, 11 pages, 2014.

[20] D. U. V. Rosenberg, Methods for Solution of Partial Differential Equations, vol. 113, Elsevier, New York, NY, USA, 1969.

[21] S. S. Siddiqi and S. Arshed, "Quintic B-spline for the numerical solution of the good Boussinesq equation," Journal of the Egyptian Mathematical Society, vol. 22, no. 2, pp. 209-213, 2014.

[22] M. Sajjadian, "Numerical solutions of Korteweg de Vries and Korteweg de Vries-Burger's equations using computer programming," International Journal of Nonlinear Science, vol. 15, no. 1, pp. 69-79, 2013. 


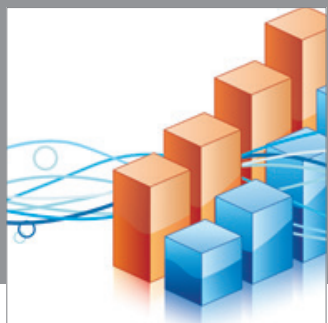

Advances in

Operations Research

mansans

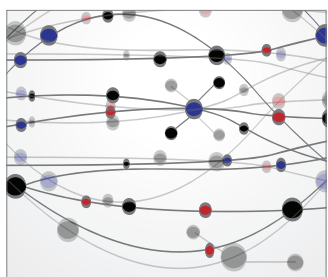

The Scientific World Journal
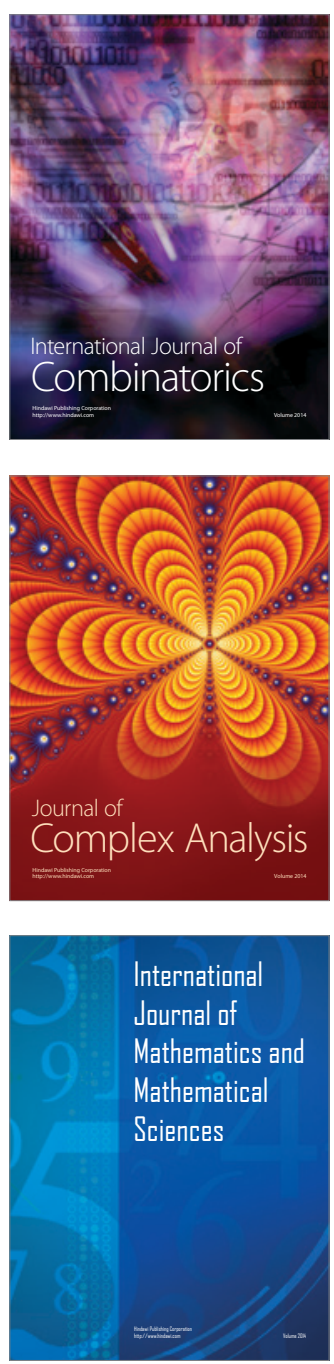
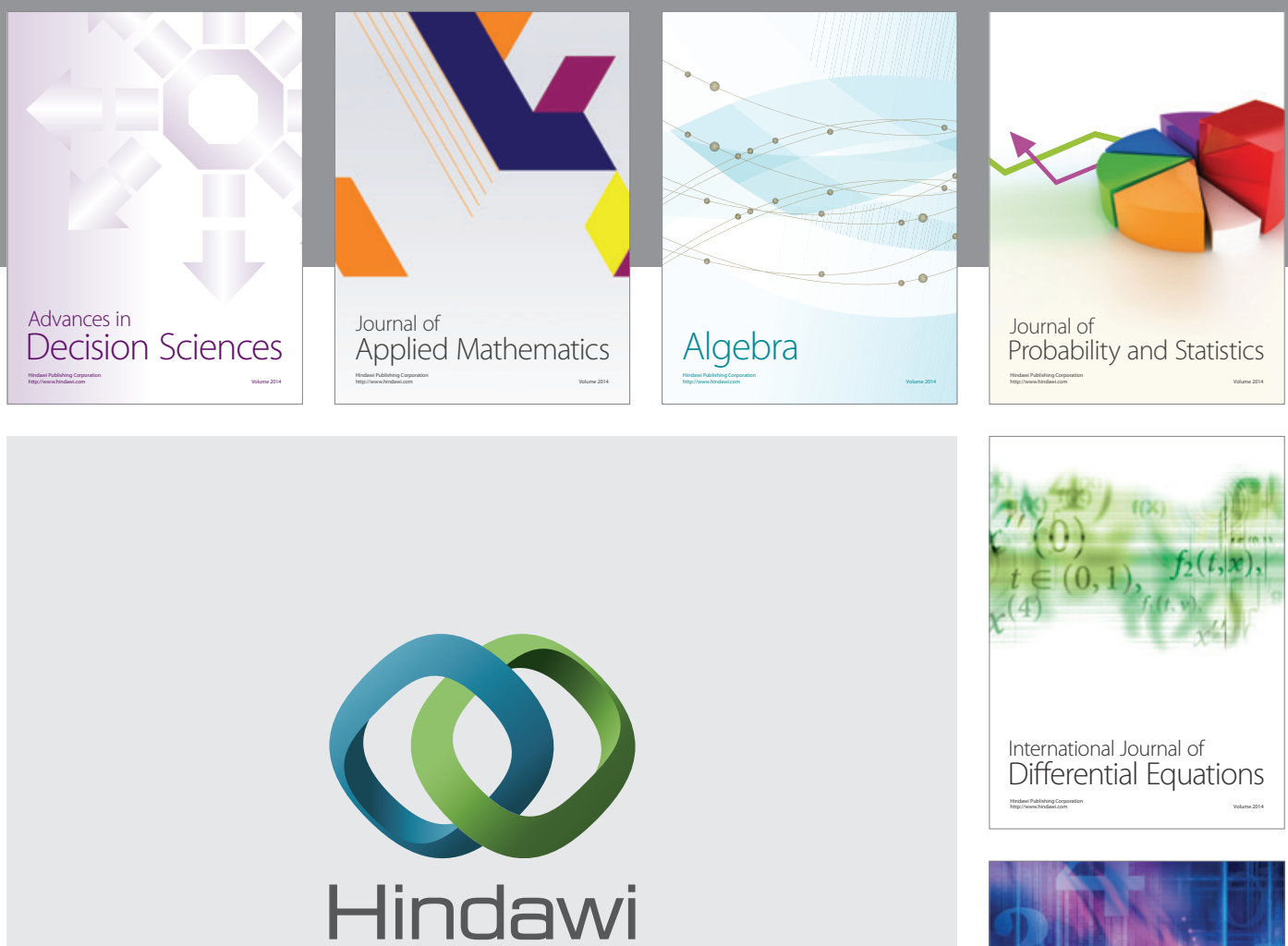

Submit your manuscripts at http://www.hindawi.com
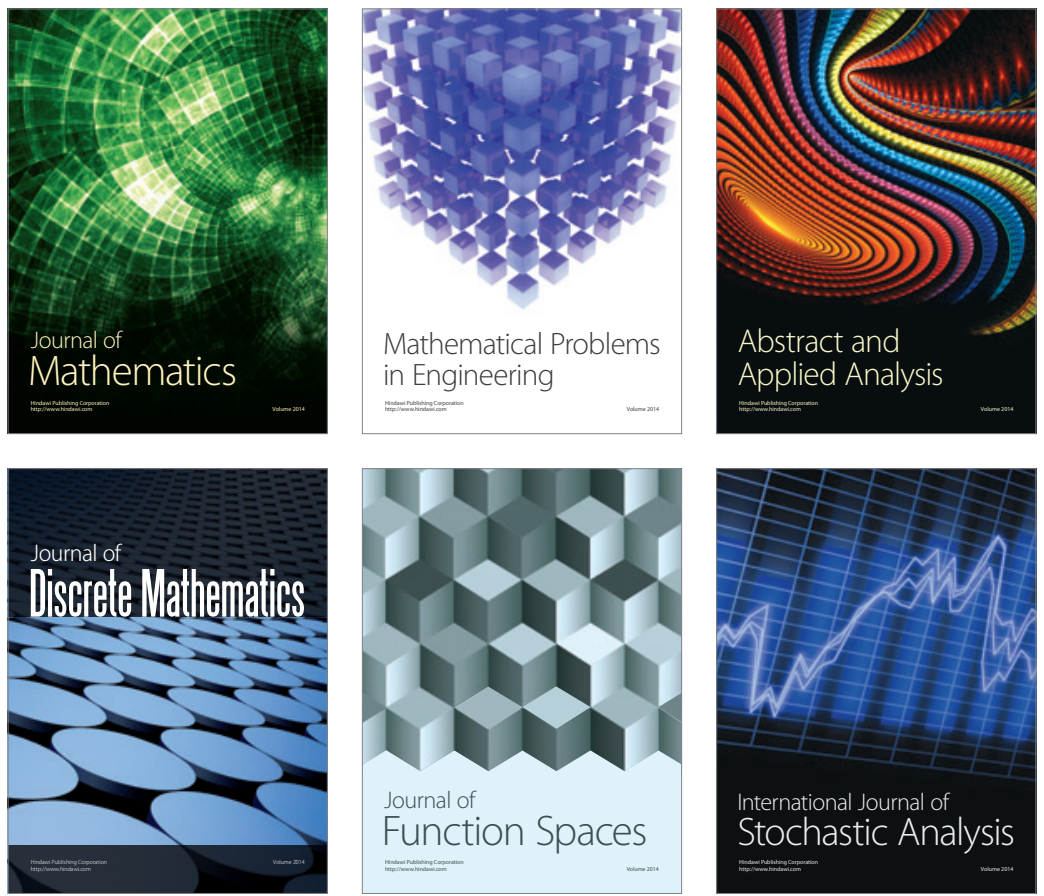

Journal of

Function Spaces

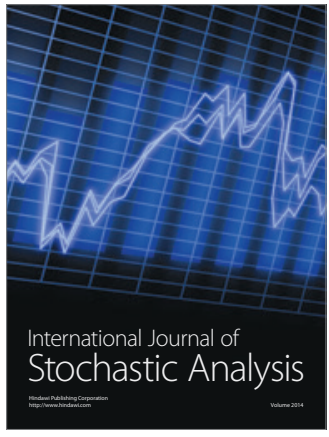

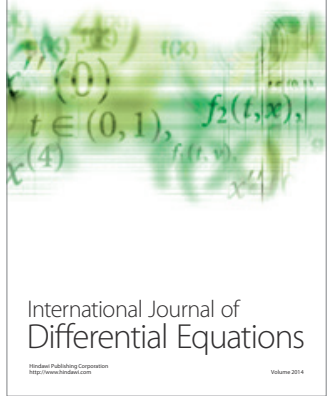
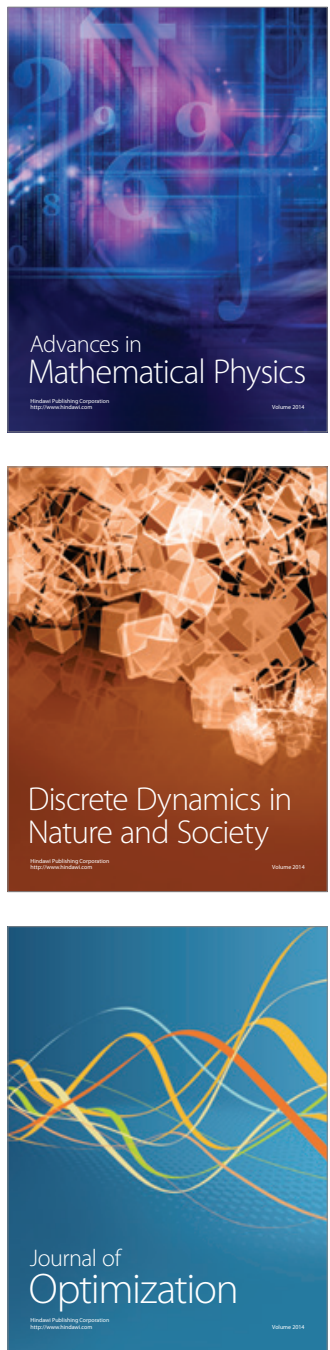